MAIN TOPIC

T. Sawai $\cdot$ R. A. Drongowski $\cdot$ R. W. Lampman

A. G. Coran · C. M. Harmon

\title{
The effect of phospholipids and fatty acids on tight-junction permeability and bacterial translocation
}

\begin{abstract}
The activity of phospholipase $\mathrm{A}_{2}\left(\mathrm{PLA}_{2}\right)$ is elevated in the intestinal epithelia of patients with inflammatory bowel disease (IBD). We recently reported that $\mathrm{PLA}_{2}$ mediates hydrolysis of phosphatidylcholine (PC) to lysophosphatidylcholine (L-PC) when both are applied to the apical surface of cultured EC monolayers, resulting in increased bacterial translocation (BT) and decreased transepithelial electrical resistance (TEER). Free fatty acids (FFA) are the other products of this reaction, however, their effect on Caco-2 cell permeability has not been reported. In addition to PC, other luminal phospholipids are present at the surface of the enterocyte. $\mathrm{PLA}_{2}$ may also mediate the hydrolysis of luminal phospholipids other than PC. The aim of this study was to examine the effects of phospholipids other than PC and common FFA on intestinal epithelial permeability and BT. Human Caco-2 enterocytes were grown to confluence on porous filters in the apical chamber of a two-chamber cell-culture system. Monolayer integrity and tight-junction permeability were measured as TEER. First, common FFA released by PC hydrolysis were determined using thin-layer chromatography (TLC). In separate experiments, monolayers were treated with phosphatidylethanolamine (PE), lysophosphatidylethanolamine (L-PE), or palmitoleic acid, oleic acids, linoleic acids, and arachidonic acid solubilized in solution with PC. The magnitude of BT was determined $2 \mathrm{~h}$ after treatment by adding Escherichia coli $C 25$ to the apical chamber followed by quantitatively culturing basal-chamber samples. Statistical analysis was by the Kurosaki-Wallis test. TLC of PC samples incubated with $\mathrm{PLA}_{2}$ on the apical surface of Caco-2 monolayers demonstrated the production of
\end{abstract}

T. Sawai · R. A. Drongowski · R. W. Lampman

A. G. Coran · C. M. Harmon ( $\bowtie)$

Section of Pediatric Surgery,

University of Michigan,

Mott Children's Hospital,

Ann Arbor, MI 48109-0245,

USA palmitoleic acid, oleic acids, linoleic acids, and arachidonic acid. L-PE significantly decreased TEER compared to controls, but to a lesser degree than L-PC alone. L-PE had no effects on BT. Palmitoleic acid and oleic acid likewise significantly decreased TEER compared to controls, however, less than L-PC. All FFA tested had no effect on BT. Phospholipids applied to the apical surface of enterocytes, such as those found in vivo in mucus, can be hydrolyzed by the enzyme PLA 2 resulting in lysophospholipid and FFA species that can alter enterocyte monolayer permeability. However, FFA and L-PL, other than L-PC, appear to have no effect to stimulate BT. This observation may have clinical implications in the pathogenesis and treatment strategies for IBD patients in whom enterocyte PLA $\mathrm{P}_{2}$ activity has been shown to be elevated.

Keywords Phospholipase $\mathrm{A}_{2} \cdot$ Phosphatidylethanolamine $\cdot$ Lysophosphatidylethanolamine $\cdot$ Free fatty acids - Bacterial translocation - Transepithelial electrical resistance $\cdot$ Inflammatory bowel disease

\section{Introduction}

Traditionally viewed as an organ of nutritional absorption, the gut also has complex defense mechanisms to prevent bacteria from entering the systemic circulation. The mucous layer is a component of the intestinal barrier, segregating potentially harmful luminal bacteria from the mucosal epithelial surface. We have previously reported that intestinal mucous phospholipid concentrations and composition are altered in neonatal, compared to adult, rabbits [13]. Phosphatidylcholine (PC), lysophosphatidylcholine (L-PC), phosphatidylethanolamine (PE), and lysophosphatidylethanolamine (L-PE) are major components of mucous phospholipids. We have also recently reported that the addition of L-PC into the apical surface promotes bacterial translocation (BT) in an enterocyte cell-culture model. 
Several human studies have documented that the activity of secretory phospholipase $\mathrm{A}_{2}\left(\mathrm{PLA}_{2}\right)$ is elevated in the intestinal mucosa and serum of patients with Crohn's disease or ulcerative colitis $[1,7-9,4]$. PLA 2 comprises a family of lipolytic enzymes that catalyze the hydrolysis of the fatty acyl ester bond at the sn-2 position of glycero-3-phospholipids to produce free fatty acids (FFA) and lysophospholipids such as L-PC. PLA 2 within the lumen of the intestine is derived from the pancreas or from mucosal Paneth-cell secretion (secretory $\mathrm{PLA}_{2}$ ). Pancreatic $\mathrm{PLA}_{2}$ functions in dietary and biliary phospholipid digestion, whereas secretory PLA $_{2}$ functions within or on the exofacial surface of intestinal mucosal epithelia. We have previously demonstrated that $\mathrm{PLA}_{2}$ mediates hydrolysis of PC to L-PC in an enterocyte cell-culture system. When both $\mathrm{PLA}_{2}$ and PC are applied to the apical surface of cultured EC monolayers, the results are increased BT and decreased transepithelial electrical resistance (TEER) [17].

In addition to hydrolyzing PC to L-PC, $\mathrm{PLA}_{2}$ in the lumen and possibly at the apical enterocyte surface may also hydrolyze other phospholipids such as PE, resulting in L-PE as well as FFA. Oleic acid and linoleic acid have been shown to induce increases in mucosal permeability in developing piglet intestine $[20,21]$. It is reported that arachidonic acid (AA), in particular, plays a role in endothelial permeability [15]. Thus, PLA 2 hydrolysis products other than L-PC on the apical surface of epithelial cells could theoretically affect mucosal permeability and BT. The purpose of this study was to test whether $\mathrm{PLA}_{2}$ hydrolysis products other than L-PC alter intestinal epithelial permeability and BT.

\section{Materials and methods}

Human colonic carcinoma (Caco-2) cells were obtained from the American Type Culture Collection No. HTB 37 (Manassas, VA). Escherichia coli $C 25$ was provided by Dr. Henri R. Ford (Pittsburgh, PA). Dulbecco's modified Eagle's medium (DMEM), fetal bovine serum, non-essential amino acid (NEAA) solution, sodium pyruvate, penicillin $G$, streptomycin, and trypsin-EDTA were purchased from Gibco (Grand Island, NY). A two-chamber cellculture system (pore size $3.0 \mu \mathrm{m}$, diameter $6.5 \mathrm{~mm}$ ), chloroform, methanol, $\mathrm{CuSO}_{4}$ pentahydrate, concentrated $\mathrm{H}_{3} \mathrm{PO}_{4}$, and MacConkey's agar were obtained from Fischer (Pittsburgh, PA). Rattail type I collagen, Dextran blue, PC, and L-PC were purchased from Sigma (St Louis, MO). Brain-heart infusion medium was obtained from BBL (Cockeysville, MD). Precoated silica gel plates (TLC plates, Silica gel 60) were purchased from EM SCIENCE (Gibbstown, NJ)

Caco- 2 cells are transformed human colon carcinoma cells that display many features of differentiated small-intestinal enterocytes. They spontaneously form polarized monolayers with tight junctions, and the apical surfaces of the cells have well-developed microvilli that contain disaccharidases and peptidases typical of normal small-intestinal villous cells. Moreover, Caco-2 cell line has been used extensively to study enterocyte interactions with bacteria such as E. coli. Cell passages 26-37 were grown on 100-mm dishes in DMEM supplemented with $10 \%$ fetal bovine serum, $1 \%$ NEAA solution, $1 \%$ sodium pyruvate, penicillin $\mathrm{G}(100 \mathrm{IU} / \mathrm{ml})$, and streptomycin $(100 \mu \mathrm{g} / \mathrm{ml})$ in a $5 \% \mathrm{CO}_{2}$ atmosphere at $37^{\circ} \mathrm{C}$. After reaching $60 \%-70 \%$ confluence, cells were harvested by trypsinization with trypsin-EDTA, washed, resuspended in DMEM and
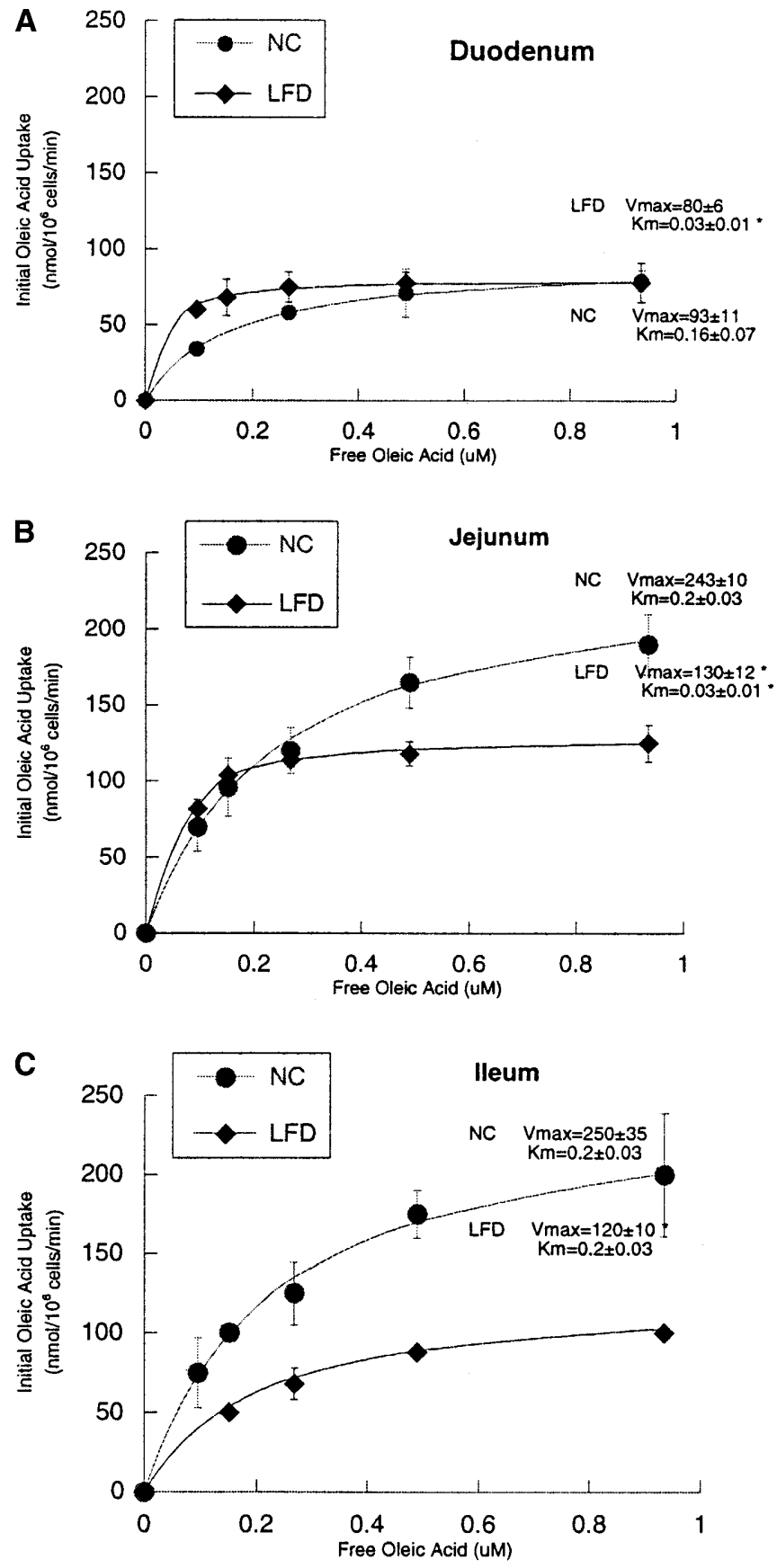

Fig. 1 Confirmation of phospholipase $\mathrm{A}_{2}\left(\mathrm{PLA}_{2}\right)$ mediated hydrolysis of phosphatidylcholine (PC) to free fatty acids: thin-layer chromatography of representative samples taken from apical Caco-2 monolayer surface treated with $\mathrm{PLA}_{2}+\mathrm{PC}(2 \mathrm{mM})$ (lane 1); standard free fatty acids; palmitoleic acids (lane 2); oleic acid (lane 3); linoleic acid (lane 4); and arachidonic acid (lane 5)

then seeded at a density of $1 \times 10^{4}$ per well $\left(0.33 \mathrm{~cm}^{2}\right)$ onto collagen-coated porous filters in the apical chamber of a two-chamber cell-culture system. Collagen coating of the plates was accomplished by incubation in $30 \mu 11.0 \mathrm{mg} / \mathrm{ml}$ rat-tail type I collagen. The cells were grown for 14 days in DMEM to reach confluence and fully differentiate. Media were changed every 2 nd day.

The tested FFA were not able to solubilize in PBS because they are hydrophobic. Although PE and L-PE are amphipathic, PE or $\mathrm{L}-\mathrm{PE}$ alone were unable to form micelles. PC, which is present in mucus, was added to the FFA, PE, and L-PE in order to solubilize 
them in PBS with micelle formation. PE and L-PE liposomes with $\mathrm{PC}$ were prepared by dissolving each phospholipid in chloroform: methanol (2:1) (CM), dried under a stream of dry nitrogen, resuspended in PBS, and subjected to cell sonication (Branson Sonifier 450; Danbury, CT).

PC was obtained from egg yolk. Thin layer chromatography (TLC) was utilized to determine which FFA to test in our cellculture system. Phospholipids were extracted using a modification of the method described by Bligh and Dyer [2]. After $120 \mathrm{~min}$ incubation of $\mathrm{PC}$ and $\mathrm{PLA}_{2}$ in the apical chamber overlying the Caco-2 monolayer, 100- $\mu$ l samples from the apical chamber were transferred to glass tubes with Teflon screw caps. Three milliliters $\mathrm{CM}$ and $700 \mu \mathrm{l}$ PBS were added and vortexed for $30 \mathrm{~s}$. The upper layer of each tube was discarded and $1.0 \mathrm{ml}$ methanol and $0.8 \mathrm{ml}$ $0.9 \% \mathrm{NaCl}$ were added and vortexed for an additional $30 \mathrm{~s}$. The upper layer of each tube was discarded by suction and the extracted lipid in the lower layer was evaporated to dryness under a stream of nitrogen and resuspended in $0.5 \mathrm{ml} \mathrm{CM}$ and subjected to TLC; $10 \mu \mathrm{l}$ of each sample was spotted on pre-coated silica gel plates. Spot separation was performed on $10 \times 20$-cm glass plates in solvent-saturated chambers. The solvent system consisted of chloroform-methanol-7N ammonia (60:35:5). After $40 \mathrm{~min}$ the plates were dried, sprayed with $8 \%(\mathrm{w} / \mathrm{v}) \mathrm{CuSO}_{4}$ pentahydrate in water/ methanol/concentrated $\mathrm{H}_{3} \mathrm{PO}_{4}(60: 32: 8)$, and charred for $15 \mathrm{~min}$ at $150{ }^{\circ} \mathrm{C}$. Each spot was recorded with a digital camera (DC 120 ZOOM, Kodak).

TEER, a measure of monolayer integrity and tight-junction permeability, was measured before and $2 \mathrm{~h}$ after addition of the following: PC alone (2 mM), PE/PC (2 mM), L-PE/PC (2 mM), PE $(1 \mathrm{mM}) / \mathrm{PC}+\mathrm{L}-\mathrm{PE}(1 \mathrm{mM}) / \mathrm{PC}$, palmitoleic acid/PC $(2 \mathrm{mM})$, oleic acid/PC $(2 \mathrm{mM})$, linoleic acid/PC $(2 \mathrm{mM})$, or AA/PC $(2 \mathrm{mM})$. TEER was measured using an epithelial voltohmmeter (EVOM; World Precision Instruments, Sarasota, FL) and expressed as the ratio of post- to pre-TEER. TEER values obtained in the absence of cells were considered as background, and resistances were calculated in $\Omega \times \mathrm{cm}^{2}$.

Bacteria that translocated across the Caco-2 monolayer were measured according to previously published methodology [6, 22] with minor modifications. Briefly, E. coli $C 25$, a nonpathogenic, streptomycin-resistant strain originally isolated from human gut flora, were grown overnight in brain-heart infusion medium, washed three times with PBS, and resuspended in PBS at a concentration of $1 \times 10^{7}$ colony forming units $(\mathrm{CFU}) / \mathrm{ml}$. The initial concentration of bacteria was determined spectrophotometrically at a wavelength of $650 \mathrm{~nm}$. Prior to addition of bacteria, the confluent Caco-2 monolayers were washed three times with DMEM without fetal bovine serum or antibiotic supplements. PC alone $(2 \mathrm{mM}), \quad$ PE/PC $(2 \mathrm{mM}), \quad$ L-PE/PC $\quad(2 \mathrm{mM}), \quad$ PE/PC $(1 \mathrm{mM})+\mathrm{L}-\mathrm{PE} / \mathrm{PC}(1 \mathrm{mM})$, palmitoleic acid/PC $(2 \mathrm{mM})$, oleic acid/PC (2 mM), linoleic acid/PC (2 mM) or AA/PC (2 mM) was added to the Caco-2 cell monolayers. After $30 \mathrm{~min}$ stabilization, E. coli $C 25$ was inoculated by adding $100 \mu \mathrm{l}$ DMEM containing $1 \times 10^{6} \mathrm{CFU}$ bacteria into the apical media. Following an additional 120 -min incubation at $37^{\circ} \mathrm{C}$ in $5 \% \mathrm{CO}_{2}$, samples from the basal chambers were taken and the number of bacterial CFU was determined by the pour-plate assay using MacConkey's agar. The magnitude of BT was expressed as $\log _{10}[\mathrm{CFU} / \mathrm{ml}]$.

Statistical analysis was performed by one-way analysis of variance. Data were expressed as mean \pm standard deviation, with statistical significance defined as $P$ less than 0.05 .

\section{Results}

\section{Thin-layer chromatography}

In order to determine which FFA are released from the hydrolysis of PC to L-PC by the enzyme PLA 2 , TLC was performed on samples taken from the apical surface of Caco-2 monolayers treated with the combination of $\mathrm{PLA}_{2}$ and $\mathrm{PC}$ at $37^{\circ} \mathrm{C}$ for $2 \mathrm{~h}$. Under our experimental conditions, palmitoleic acid, oleic acid, linoleic acid, and AA were generated (Fig. 1).

\section{Effect on TEER}

Linoleic acid (2 $\mathrm{mM})$ and AA (2 $\mathrm{mM})$ solubilized in PC micelles had no effect on TEER compared to PC alone or PBS as controls. However, palmitoleic acid $(2 \mathrm{mM})$ and oleic acid $(2 \mathrm{mM})$ resulted in a small but significant decrease in TEER compared to PC or PBS. TEER was decreased $94 \%$ by L-PC, $23 \%$ by palmitoleic acid, and $21 \%$ by oleic acid (Fig. 2). Interestingly, AA and PE did not alter TEER. As expected, PC alone resulted in no significant change in TEER compared to cell monolayers treated with PBS alone. Also as expected, L-PC
Fig. 2 Effects of palmitoleic acid, oleic acid, linoleic acid, and arachidonic acid on transepithelial electrical resistance (TEER) across Caco-2 monolayers. TEER was measured before and $2 \mathrm{~h}$ after addition of free fatty acids. Data expressed as the mean $\pm \mathrm{SD}(P C$ phosphatidylcholine, $L-P C$ lysophosphatidylcholine, $P B S$ phosphate-buffered saline). $* P<0.05$ vs PBS and PC; ** $P>0.05$ vs PBS and PC

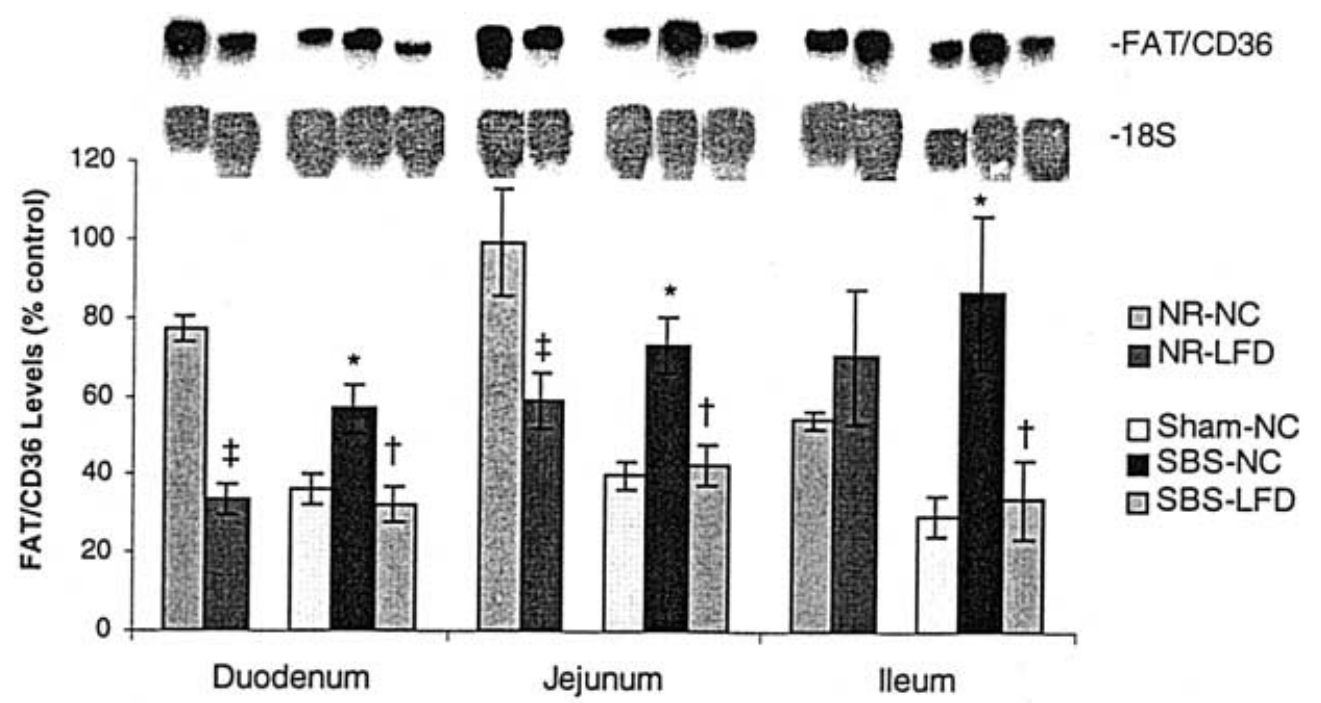

Bowel Segments 

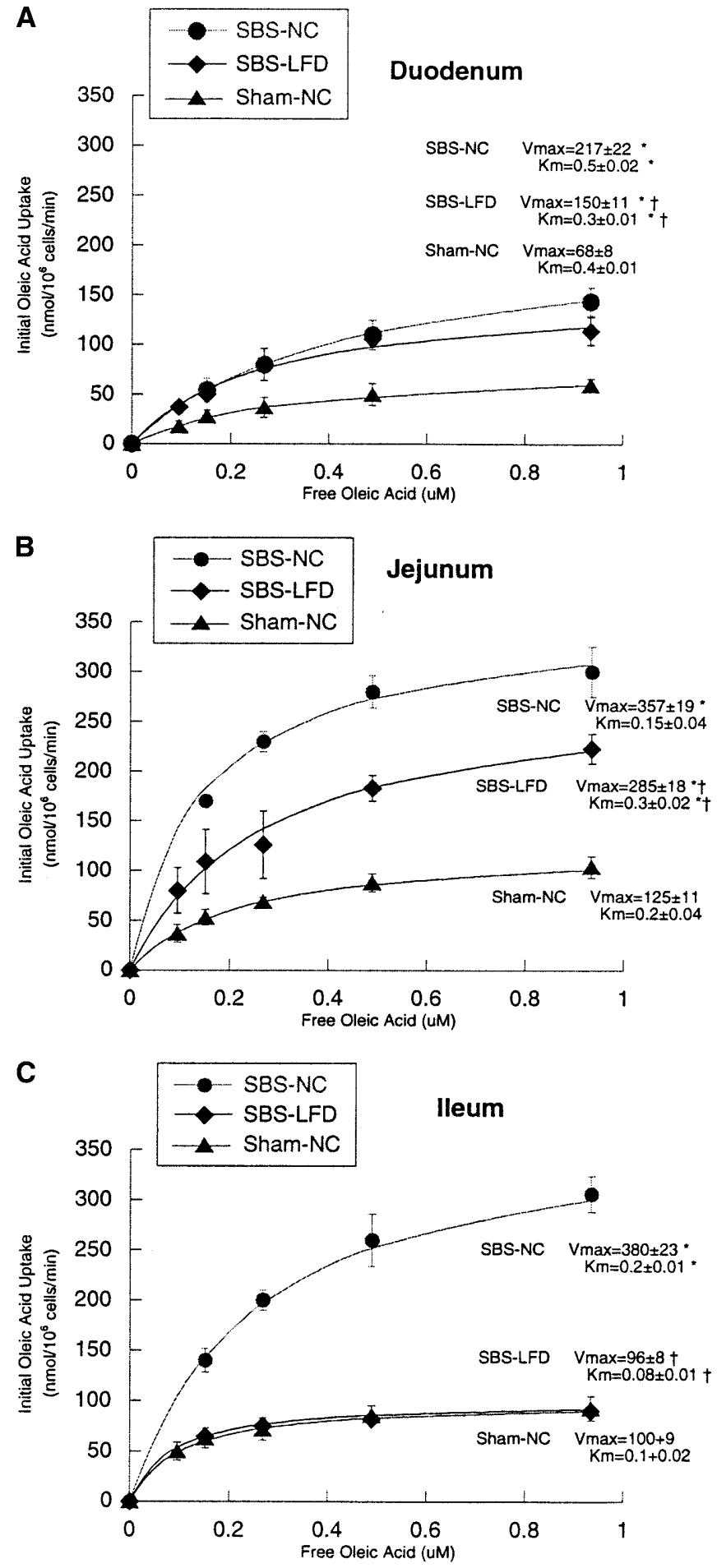

Fig. 3 Effects of phosphatidylethanolamine (PE), lyso-PE (L-PE), and PE + L-PE on transepithelial electrical resistance across Caco-2 monolayers. Bacterial translocation was measured $2 \mathrm{~h}$ after addition of PE and/or L-PE. Data expressed as mean \pm SD ( $P C$ phosphatidylcholine, $L-P C$ lyso-PC, $P B S$ phosphate-buffered saline).

$* P<0.05$ vs PBS and PC; $* * P<0.05$ vs PBS

significantly decreased TEER compared to PBS and PC by $94 \%$ and $93 \%$ respectively. Treatment of these monolayers with L-PE significantly decreased TEER compared to PC $(30 \%)$ or PBS $(24 \%)$ as a control, but

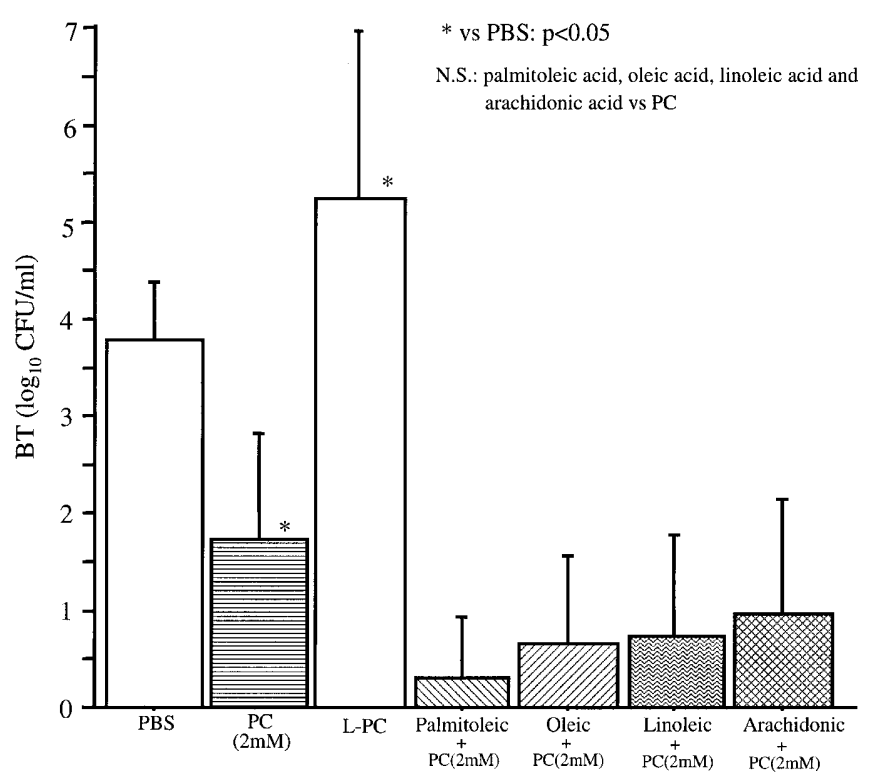

Fig. 4 The effect of palmitoleic acid, oleic acid, linoleic acid, and arachidonic acid on bacterial translocation (BT) (expressed as $\left.\log _{10}[\mathrm{CFU} / \mathrm{ml}]\right)$ across Caco-2 monolayers. BT was measured $2 \mathrm{~h}$ after addition of free fatty acids. Data expressed as mean $\pm \mathrm{SD}(P C$, phosphatidylcholine, $L-P C$ lyso-PC, $P B S$ phosphate-buffered saline)

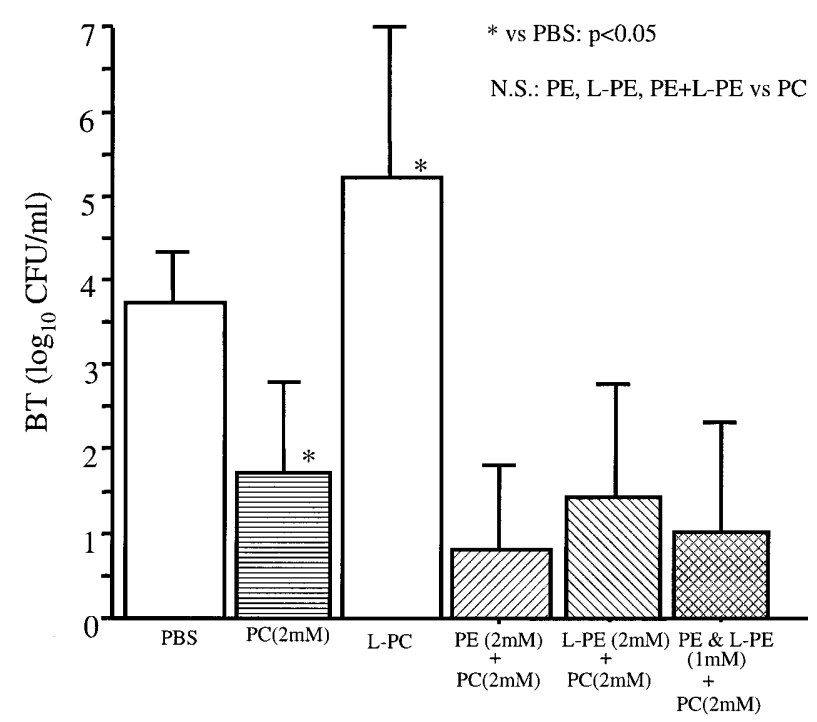

Fig. 5 Effect of phosphatidylethanolamine $(P E)$ and/or lyso-PE $(L-P E)$ on bacterial translocation $(B T)$ (expressed as $\left.\log _{10}[\mathrm{CFU} / \mathrm{ml}]\right)$ across Caco-2 monolayers. BT was measured $2 \mathrm{~h}$ after addition of free fatty acids. Data expressed as mean $\pm \mathrm{SD}(P C$ phosphatidylcholine, $L-P C$ lyso-PC, $P B S$ phosphate-buffered saline)

the decrease was less dramatic than for L-PC alone (94\%) (Fig. 3).

\section{Effect on BT}

As previously presented, L-PC increased BT and PC decreased BT compared to PBS. However, none of the 
FFA solubilized in the PC micelle had a significant effect on BT compared to PC (Fig. 4). Neither L-PE nor PE had a significant effect on BT compared to PC (Fig. 5).

\section{Discussion}

The cause of inflammatory bowel disease (IBD) remains unknown. Current information suggests that either bacteria or various nutrients in the intestinal lumen act as antigens, thereby activating $\mathrm{T}$ cells or macrophages $[3,19]$. It is generally believed that IBD may result from the host being exposed to an excessive or long-term state of inflammation. In this state, it has been demonstrated that the activity of secretory $\mathrm{PLA}_{2}$ is elevated in the intestinal mucosa and serum of patients with IBD [1,7$9,14]$. Secretory $\mathrm{PLA}_{2}$ is only present in the Paneth cells in normal intestinal mucosa [11]. However, under conditions of IBD it is generated by both Paneth and epithelial cells [4]. Interestingly, sulfasalazine, a drug widely used as a therapy in several inflammatory conditions such as rheumatoid arthritis and IBD, is believed to act via inhibition of the extracellular release of pro-inflammatory secretory $\mathrm{PLA}_{2}[16]$.

We have previously demonstrated that L-PC converted from PC with $\mathrm{PLA}_{2}$ at the apical surface of an enterocyte cell-culture model increases permeability and BT [17]. Neither PC nor PLA $\mathrm{P}_{2}$ alone has any effect on permeability or $\mathrm{BT}$ in this model. Alterations in the composition of the mucous layer may promote BT under pathological conditions such as gut immaturity [18]. Therefore, we have hypothesized that the elevation of $\mathrm{PLA}_{2}$ activity in the intestinal mucosa of patients with IBD might lead to increased permeability and BT. In this study, we demonstrated that neither PE, L-PE, palmitoleic acid, linoleic acid, oleic acid, or AA, which are present in the mucous layer of our cell-culture model, had an affect on TEER or BT when compared to L-PC. However, when $\mathrm{PLA}_{2}$ is elevated, its activity on PC conversion to L-PC may cause an increase in mucosal permeability and subsequent BT.

The mechanism by which PLA $_{2}$ activity in the mucosa of patients with IBD is elevated is still unclear. Stimulation of the cytokines interleukin (IL)-1, IL-6, tumor-necrosis factor-alpha, or interferon- $\gamma$, which have reportedly been increased in IBD, may induce these inflammation states $[3,5,10,12,19]$. This may lead to an excess of secretory $\mathrm{PLA}_{2}$. PC, which is found in vivo in the mucous intestinal layer, applied to the apical surface of an enterocyte monolayer cell culture can be hydrolyzed by the enzyme PLA $\mathrm{P}_{2}$, resulting in lysophospholipid and FFA species that can alter monolayer permeability. However, other FFA and phospholipids present in the mucous layer appear to have no effect on BT. This observation may have clinical implications in the pathogenesis and treatment strategies for IBD patients in whom enterocyte $\mathrm{PLA}_{2}$ activity has been shown to be elevated.

\section{References}

1. Almer S, Franzen L, Olaison G, Smedh K, Strom M (1991) Phospholipase $A_{2}$ activity of colonic mucosa in patients with ulcerative colitis. Digestion 50: 135-141

2. Bligh E, Dyer W (1959) A rapid method of total lipid extraction and purification. Can J Biochem Physiol 37: 911-917

3. Fiocchi C (1998) Inflammatory bowel disease: etiology and pathogenesis. Gastroenterology 115: 182-205

4. Haapamaki MM, Gronroos MJ, Nurmi H, Alanen K, Nevalainen JT (1999) Gene expression of group II phospholipase $\mathrm{A}_{2}$ in intestine in Crohn's disease. Am J Gastroenterol 94: 713-720

5. Holtkamp W, Stollberg T, Reis HE (1995) Serum interleukin-6 is related to disease activity but not disease specificity in inflammatory bowel disease. J Clin Gastroenterol 20: 123-126

6. Kops S, Lowe D, Bement W, West A (1996) Migration of Salmonella typhi through intestinal epithelial monolayers: an in vitro study. Microbiol Immunol 40: 799-811

7. Minami T, Tojo H, Shinomura Y, Tarui S, Okamoto M (1992) Raised serum activity of phospholipase $\mathrm{A}_{2}$ immunochemically related to group II enzyme in inflammatory bowel disease: its correlation with disease activity of Crohn's disease and ulcerative colitis. Gut 33: 914-921

8. Minami T, Tojo H, Shinomura Y, Komatsubara T, Matsuzawa Y, Okamoto M (1993) Elevation of phospholipase $A_{2}$ protein in sera of patients with Crohn's disease and ulcerative colitis. Am J Gastroenterol 88: 1076-1080

9. Minami T, Tojo H, Shinomura Y, Matsuzawa Y, Okamoto M (1994) Increased group II phospholipase $A_{2}$ in colonic mucosa of patients with Crohn's disease and ulcerative colitis. Gut 35: $1593-1598$

10. Murch SH, Lamkin VA, Savage MO, Walker Smith JA, MacDonald TT (1991) Serum concentrations of tumor necrosis factor alpha in childhood chronic inflammatory disease. Gut 32: 913-917

11. Nevalainen JT, Gronroos MJ, Kakkajoki M (1995) Expression of group II phospholipase $\mathrm{A}_{2}$ in the human gastrointestinal tract. Lab Invest 72: 201-208

12. Niessner M, Volk BA (1995) Altered Th1/Th2 cytokine profiles in the intestinal mucosa of patients with inflammatory disease as assessed by quantitative reversed transcribed polymerase chain reaction (RT-PCR). Clin Exp Immunol 101: 428-435

13. Okuyama H, Urao M, Lee D, Abe A, Drongowski R, Harmon C, Coran A (1998) Changes, with age, in the phospholipid content of the intestinal mucus layer of the newborn rabbit. J Pediatr Surg 33: 31-38

14. Olaison G, Leandersson P, Sjeodahl R, Tagesson C (1989) Increased in permeability and phospholipase $\mathrm{A}_{2}$ activity of colonic mucosa in Crohn's colitis. Digestion 43: 228-233

15. Onishi T, Posner B, Shapiro R (1992) Vasogenic brain edema induced by arachidonic acid: role of extracellular arachidonic acid in blood-brain barrier dysfunction. Neurosurgery 30: 545551

16. Pruzanski W, Stefanski E, Vadas P, Ramamurthy SN (1997) Inhibition of extracellular release of proinflammatory secretory phospholipase $\mathrm{A}_{2}\left(\mathrm{sPLA}_{2}\right)$ by sulfasalazine: a novel mechanism of anti-inflammatory activity. Biochem Pharmacol 53: 19011907

17. Sawai T, Usui N, Dwaihy J, Drowgowski R, Abe A, Coran A, Harmon C (2000) The effect of phospholipase $A_{2}$ on bacterial translocation in a cell culture model. Pediatr Surg Int 16: 262 266

18. Usui N, Ray CJ, Drongowski RA, Coran AG, Harmon CM (1999) The effect of phospholipids and mucin on bacterial internalization in an enterocyte cell culture model. Pediatr Surg Int 15: $150-154$

19. Van Dullemen H, Meenan J, Stronkhorst A, Tytgat G, Van Deventer S (1997) Mediators of mucosal inflammation: implications for therapy. Scand J Gastroenterol 32 [Suppl 223]: 92-98 
20. Velasquez R, Henninger K, Fowler M, Tso P, Crissinger K (1993) Oleic acid-induced mucosal injury in developing piglet intestine. Am J Physiol 264: G576-G582

21. Velasquez R, Tso P, Crissinger K (1993) Fatty acid-induced injury in developing piglet intestine: effect of degree of saturation and carbon chain length. Pediatr Res 33: 543-547
22. Wells C, Jechorek R, Erlandsen S (1995) Inhibitory effect of bile on bacterial invasion of enterocytes: possible mechanism for increased translocation associated with obstructive jaundice. Crit Care Med 23: 301-307 